\title{
MODELO ORIENTADO A OBJETOS PARA AVALIAÇÃO DA CONFIABILIDADE COMPOSTA POR SIMULAÇÃO MONTE CARLO COM REPRESENTAÇÃO DA GERAÇÃO EÓLICA
}

\author{
Júlio Alberto Silva Dias* \\ julio.dias@eletrobras.com
}

\author{
Carmen Lucia Tancredo Borges ${ }^{\dagger}$ \\ camen@nacad.ufrj.br
}

\author{
*Eletrobrás - Centrais Elétricas Brasileiras S.A. \\ Av. Mal Floriano, 14, Rio de Janeiro, RJ \\ ${ }^{\dagger}$ COPPE/UFRJ - Programa de Engenharia Elétrica \\ Caixa Postal 68504 - CEP 21941-972 - Rio de Janeiro - RJ
}

\section{RESUMO}

Este trabalho apresenta um modelo computacional flexível orientado a objetos para avaliação da confiabilidade composta de sistemas de potência por Simulação Monte Carlo Seqüencial e Não-Seqüencial. O enfoque principal dado ao modelo foi a representação de grandezas variantes no tempo, tais como a geração eólica e a curva de carga. A flexibilidade da modelagem permite a representação de componentes de rede complexos, além da incorporação de modelos de simulação híbridos que combinem características da simulação sequencial e da não-sequencial. Os resultados obtidos para vários estudos baseados no sistema IEEE-RTS comprovam a aplicabilidade do modelo desenvolvido e permitem levantar várias conclusões sobre a influência da geração eólica nos índices de confiabilidade do sistema.

PALAVRAS-CHAVE: Confiabilidade Composta, Modelagem Orientada a Objetos, Simulação Monte Carlo, Geração Eólica.

\section{ABSTRACT}

This paper presents a flexible object-oriented computational model for composite systems reliability evaluation based on

Artigo submetido em 21/09/2008 (Id.: 00897)

Revisado em 03/03/2009, 31/03/2009

Aceito sob recomendação do Editor Associado Prof. Eduardo N. Asada
Sequential and Non-Sequential Monte Carlo simulation. The main focus of the model is in the representation of time varying components, such as the wind generation and the load. The modeling flexibility enables the representation of complex network components, as well as the incorporation of hybrid simulation approaches that combine sequential and nonsequential characteristics. The results obtained with several studies based on the IEEE RTS system prove the applicability of the developed model and provide important conclusions about wind generation influence on power system reliability indices.

KEYWORDS: Composite Reliability, Object Oriented Modeling, Monte Carlo Simulation, Wind Generation.

\section{INTRODUÇÃO}

A natureza complexa dos sistemas de potência requer que grandes sistemas computacionais sejam desenvolvidos para auxiliar nas atividades de planejamento e operação. Os softwares disponíveis para o setor elétrico são, em sua maioria, escritos em linguagens convencionais, baseadas na primeira geração das linguagens de alto nível. Os softwares escritos nestas linguagens costumam ser de difícil manutenção, atualização e reutilização de código, o que resulta em grandes investimentos para as modificações, apresentando ainda um baixo nível de integração com outros softwares. 
A Modelagem Orientada a Objetos (MOO), como metodologia de desenvolvimento de softwares, apresenta-se como uma opção promissora para enfrentar os novos desafios da produção de ferramentas computacionais para o setor elétrico. A MOO visa a construção de uma estrutura de dados sólida e consistente, a partir da qual são implementados softwares flexíveis, com alto grau de reutilização dos códigos e com facilidades para manutenção e atualização.

Este trabalho apresenta um modelo computacional flexível orientado a objetos para avaliação da confiabilidade composta de sistemas de potência por Simulação Monte Carlo Seqüencial e Não-Seqüencial. A flexibilidade da modelagem permite a representação precisa de elementos complexos, como os dispositivos FACTS, e a representação de fenômenos variantes no tempo. Com isto, tanto a geração eólica quanto a curva de carga podem ser adequadamente modeladas na avaliação da confiabilidade composta.

Trabalhos publicados abordam a representação de geração eólica na avaliação da confiabilidade composta sob enfoques distintos. Alguns trabalhos são baseados em Simulação Monte Carlo Sequencial, tais como (Billinton et al., 1996), (Karki e Billinton, 2001) e (Billinton e Bai, 2004), onde o comportamento do vento é modelado de forma cronológica para avaliação da geração eólica. Outros trabalhos utilizam a representação da geração por modelo a múltiplos estados, que podem ser utilizados na Simulação Monte Carlos NãoSequencial. Em (Singh e Kim, 1988) é proposto um método de representação da geração eólica a partir da clusterização dos estados da geração. Em (Karki e Hu, 2005) é proposto um modelo de múltiplos estados para usinas eólica, sendo concluído que são necessários pelo menos 7 estados de geração para uma representação adequada. Em (Leite et al., 2006a-b) é proposto um modelo de múltiplos estados para usina eólica com flexibilidade para representar os tipos de controles das turbinas e a variação das taxas de falha e reparo das turbinas de acordo com o estado do vento. Em (Billinton e Gao, 2008) é aplicado um modelo de múltiplos estados para representação da usina eólica na Simulação Monte Carlo Não-Seqüencial, sendo considerado até 11 estados de geração.

Em relação a representação da variação da carga na avaliação da confiabilidade composta, alguns trabalhos podem ser destacados. Em (Melo et al., 1992) é proposto o modelo para representar múltiplos estados individuais de carga desbalanceados em freqüência, baseados em modelos de Markov e obtidos a partir da curva cronológica de carga, utilizando técnicas de agrupamento. Em (Sankarakrishnan et al., 1995) uma curva cronológica anual é adotada para cada barra de carga do sistema, sendo os índices de confiabilidade obtidos por simulação Monte Carlo seqüencial. Em (Mello et al., 1997) é apresentada a simulação pseudo-sequencial com re- presentação Markoviana, na qual a hipótese Markoviana é adotada para todo sistema, inclusive a carga. Em (Leite da Silva et al., 2000) é sugerida uma variação desta metodologia, onde a carga é representada por um modelo de Markov não-agregado com múltiplos níveis. Em (Manso e Leite da Silva, 2004) este modelo de carga é utilizado em uma simulação Monte Carlo Não-Sequencial.

Em relação a aplicação de Modelagem Orientada a Objetos em sistemas de potência, alguns trabalhos reportam o desenvolvimento de ambientes e aplicativos específicos. Em (Neyer et al., 1990) a orientação a objetos é aplicada para representação de elementos da rede na solução do fluxo de potência. Em (Hakavik e Holen, 1994) são utilizados os conceitos inerentes à MOO para demonstrar as facilidade de aplicação das técnicas de esparsidade. Em (Foley e Bose, 1995) é sugerida uma estrutura computacional para representar a topologia física da rede, reproduzindo as características reais do sistema. Em (Fuerte-Esquivel et al., 1998) é proposto um modelo orientado a objetos para estudos de sistemas de grande porte contendo dispositivos FACTS. Em (Manzoni et al., 1999) é apresentada uma estrutura orientada a objetos para aplicações em simulações dinâmicas, em que se tem uma classe principal do sistema elétrico e outras classes derivadas para representar os elementos físicos e topológicos da rede. Em (Manzoni, 2005), esta estrutura orientada a objetos é expandida, resultando no ambiente utilizado neste trabalho como base para o desenvolvimento do aplicativo de avaliação da confiabilidade composta.

Diferentemente das referências anteriores, este trabalho apresenta a aplicação de MOO no desenvolvimento de um modelo para avaliação da confiabilidada composta que agrega os dois enfoques da simulação Monte Carlo e a representação tanto da geração eólica como da carga variante. Para tanto, são propostas formas de representação da variação da geração eólica e da carga tanto na simulação sequencial como na não-sequencial. O principal objetivo deste trabalho é apresentar uma ferramenta flexível que pode ser expandida com facilidade para incluir modelos de componentes de rede complexos e incorporar modelos de simulação híbridos que combinem características da simulação sequencial e da nãosequencial.

\section{CONFIABILIDADE COMPOSTA}

A avaliação da confiabilidade composta de sistemas de potência, sob o enfoque da adequação, visa avaliar se o sistema é capaz de atender à carga em seus diversos pontos de consumo, estando sujeito a ocorrência de falhas nos sistemas de geração e transmissão. Para tanto, são calculados índices de confiabilidade, tais como LOLP (Probabilidade de Perda de Carga), EPNS (Expectância de Potência Não Suprida), LOLF (Frequência de Perda de Carga), etc. 
O sistema de potência é composto por um conjunto de componentes, tais como geradores, cargas, transformadores, linhas de transmissão, entre outros. Um estado do sistema consiste da combinação dos estados de cada um destes componentes. Processos de Markov têm sido utilizados para modelar o comportamento estocástico de componentes do sistema, através de modelos a 2 ou múltiplos estados ou através do tratamento de séries temporais.

A avaliação da confiabilidade composta pode ser realizada por meio de duas representações distintas do sistema: espaço de estados e representação cronológica. Na representação por espaço de estados, os estados do sistema podem ser selecionados através de duas técnicas: enumeração de estados e Simulação Monte-Carlo Não Seqüencial. Já na representação cronológica, os estados dos componentes são sequencialmente amostrados por vários períodos de tempo, simulando a cronologia do processo estocástico de operação do sistema. A técnica para representação cronológica mais utilizada é a Simulação Monte Carlo Seqüencial.

\subsection{Simulação Monte Carlo Seqüencial}

Na Simulação Monte Carlo Seqüencial (SMC Seqüencial) os estados amostrados preservam as características cronológicas do sistema, existindo a preocupação de se amostrar corretamente os estados consecutivos do sistema, bem como seguir as curvas de carga e geração, quando existentes. Teoricamente, a simulação Monte Carlo Seqüencial é a metodologia que produz os resultados mais reais na avaliação da confiabilidade composta, sendo comumente adotada como referência para validação de outras metodologias.

O cálculo dos índices de confiabilidade pode ser expresso pela avaliação da expressão (1):

$$
\bar{E}(G)=\frac{1}{N} \sum_{k=1}^{N} G\left(y_{k}\right)
$$

onde $N$ é o número de séries sintéticas anuais simuladas, $y_{k}$ é a série sintética anual composta pelos estados amostrados seqüencialmente dentro do ano $k, G$ é uma função teste para o cálculo dos índices de confiabilidade anuais e $\bar{E}(G)$ fornece uma estimativa dos índices de confiabilidade calculados sobre todas as séries sintéticas simuladas.

Em (Borges et al., 2001) é sugerido um algoritmo conceitual para avaliação da confiabilidade composta a partir da SMC Seqüencial:

1. Gere uma série sintética anual de estados do sistema $y_{k}$ , amostrando-se sequencialmente no tempo os estados dos componentes;
2. Analise a adequação do estado $\underline{x}$ da série $y_{k}$ a partir de uma função de avaliação $F(\underline{x})$ e acumule os resultados relativos ao ano $k$;

3. Calcule os índices de confiabilidade anuais relativos ao ano pela avaliação da função $G\left(y_{k}\right)$ sobre os valores acumulados no passo 2;

4. Atualize a estimativa de $C(G)$, o valor esperado dos índices de confiabilidade do processo, baseado no resultado obtido no passo 3,

5. Se a precisão das estimativas é aceitável, o processo é dito convergido, caso contrário retorne ao passo 1.

A série sintética anual do passo 1 do algoritmo é gerada pela combinação dos processos de transição de estados dos componentes do sistema e da variação cronológica dos modelos variantes no tempo. O processo de transição de estados dos componentes é obtido pela amostragem seqüencial da distribuição de probabilidade da duração dos estados.

\subsection{Simulação Monte Carlo Não- Seqüencial}

Na Simulação Monte Carlo Não-Seqüencial (SMC NãoSeqüencial) os estados do sistema são selecionados por amostragem do espaço de estados, sem preocupação com a cronologia dos eventos. Cada componente do sistema possui um modelo estocástico que descreve os seus possíveis estados e probabilidades de operação.

O cálculo dos índices de confiabilidade pode ser expresso pela avaliação da expressão (2):

$$
\bar{E}(F)=\frac{1}{N} \sum_{i=1}^{N} F\left(\underline{x}^{i}\right)
$$

onde $N$ é o número de estados simulados, $F$ é uma função teste para o cálculo dos índices de confiabilidade para cada estado do sistema $\underline{x}^{i}$ e $\bar{E}(F)$ fornece uma estimativa dos índices de confiabilidade anuais.

Em (Pereira e Balu, 1992) é sugerido um algoritmo conceitual para avaliação da confiabilidade composta a partir da SMC Não-Sequiencial:

1. Selecione um estado do sistema $\underline{x}$ ? X correspondente a um ponto de operação, isto é, disponibilidade de componentes, níveis de carga e geração, etc;

2. Analise a adequação do estado $\underline{x}$ a partir de uma função de avaliação $F(\underline{x})$; 
3. Atualize a estimativa $C(F)$, o valor esperado dos índices de confiabilidade do processo, baseado no resultado obtido no passo 2;

4. Se a precisão das estimativas é aceitável, o processo é dito convergido, caso contrário retorne ao passo 1.

Na abordagem não-seqüencial, os estados do sistema selecionados no passo 1 do algoritmo dependem da combinação dos estados de todos os seus componentes, sendo estes determinados pela amostragem da distribuição de probabilidade de estados de cada componente.

A principal dificuldade da abordagem não seqüencial está no cálculo dos índices de freqüência e duração. A rigor, para determinação do índice LOLF, para cada estado $i$ de corte do sistema, seria necessário identificar todos os estados sem corte para quais ele pode transitar. Para um sistema com $m$ componentes modelados a 2 estados, seriam necessárias $m$ análises de adequação adicionais. No caso de componentes modelados a mais estados, este problema se torna ainda mais complexo. Na literatura existem algumas propostas para o cálculo deste índice com um menor esforço computacional, destacando-se o método da probabilidade condicional (Mello et al., 1993), que não exige nenhuma análise de adequação adicional além das já necessárias para cálculo dos índices LOLP e EPNS. A restrição deste método é em relação a suposição de que o sistema é coerente, isto é, supor que se um componente falho é reparado, o desempenho do sistema nunca piora, ou se um componente falhar, o desempenho do sistema nunca melhora.

O fator favorável à utilização da simulação não-seqüencial ao invés da seqüencial é o menor esforço computacional exigido, sendo a principal limitação o fato de se perder os aspectos cronológicos da simulação, que em alguns estudos podem ser relevantes.

\subsection{Análise de Adequação dos Estados}

A análise de adequação dos estados é a etapa que demanda o maior esforço computacional no processo de simulação. Esta análise verifica se o sistema, no estado em que foi amostrado, é capaz de atender à demanda de energia sem violar os limites operativos e/ou cortar carga. Basicamente, a análise de adequação consiste em verificar se as condições de operação estão sendo atendidas, ou se é necessário alguma medida corretiva a fim de tentar evitar o corte de carga. Estas medidas corretivas requerem a solução de um problema de otimização, que deve considerar ações de redespacho de geração, acionamento de compensadores ou qualquer outra medida possível na operação do sistema. Desta forma, para esta etapa, é necessário a solução de um problema de fluxo de potência não linear (Monticelli, 1983), seguido da solução de um fluxo de potência ótimo não linear, com flexibilidade suficiente para representação dos mais variados tipos de controles.

\section{GRANDEZAS VARIANTES NO TEMPO}

\subsection{Cargas}

\subsubsection{Modelo Seqüencial}

O modelo da carga para aplicação na SMC Seqüencial é a cruva cronológica de variação da carga. Sua consideração depende do nível de discretização da curva de carga e do passo de simulação, que são em geral horários. Em um determinado instante de simulação $t_{k}$, para cada ponto de carga $L_{i}$ pertencente a uma determinada área $n$, a demanda $P_{L i}$ pode ser calculada como sendo:

$$
P_{\text {Li }}\left(t_{k}\right)=P_{\text {Linom }} \cdot A_{n}\left(t_{k}\right)
$$

onde $P_{\text {Linom }}$ expressa o valor da potência nominal do ponto de carga $i$ e $A_{n}(t)$ é a curva cronológica de carga expressa em pu.

No modelo seqüencial, a consideração de diferentes curvas de carga por área ou barra do sistema pode ser feita de forma direta, apenas considerando o valor das cargas em cada passo de simulação. Na Figura 1 é apresentada uma representação em blocos para o modelo implementado, onde para efeito de facilidade de ilustração, são considerados dois pontos com a mesma curva de carga.

A combinação das séries temporais de cada carga com as séries sintéticas amostradas para os outros componentes gera a série sintética anual do sistema.

\subsubsection{Modelo Não-Seqüencial}

Uma abordagem comum para a representação da carga na SMC Não-Sequiencial é a adoção de um único modelo Markoviano a múltiplos estados para representar a carga em todo o sistema, sendo que cada barra responde por um percentual deste total. Isso corresponde a considerar as cargas das barras totalmente correlacionadas com a carga do sistema. Este modelo possui restrições relacionadas à impossibilidade de se representar os aspectos regionais de cada ponto de carga (Manso e Leite, 2004). No entanto, não é objetivo deste trabalho um maior aprofundamento na questão de ter-se diferentes curvas de carga por área e, desta forma, o modelo de carga adotado é o modelo Markoviano agregado único para todo o sistema. O modelo sequiencial equivalente seria a consideração de uma única área com uma única curva de carga para todas as barras do sistema na Figura 1. 


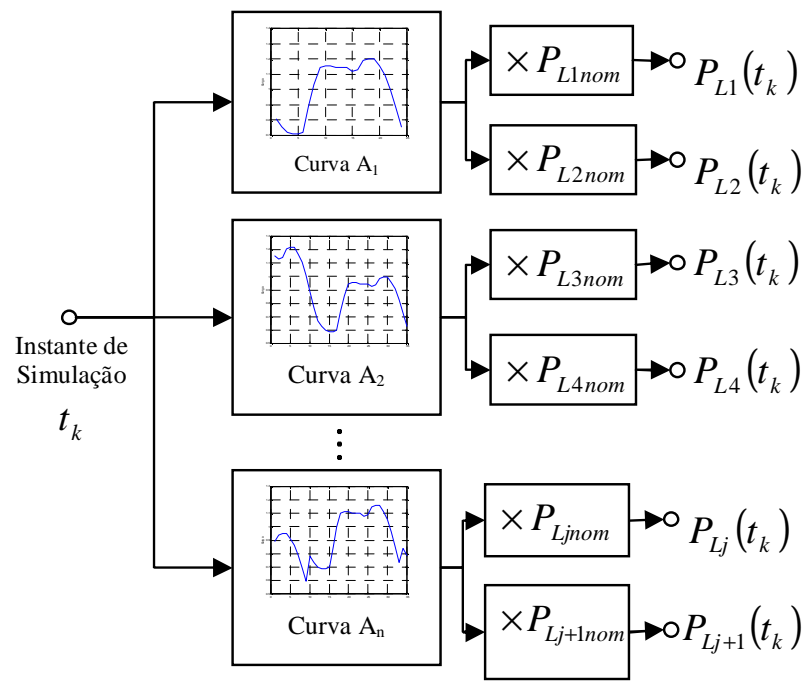

Figura 1: Modelo de Carga Cronológica

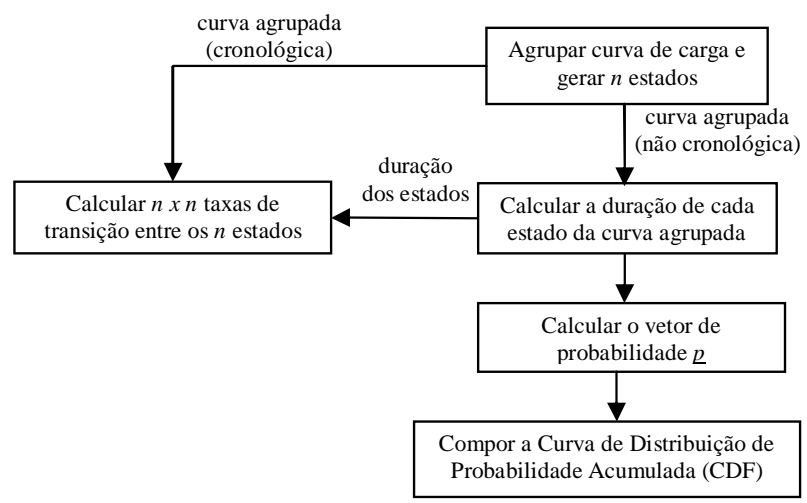

Figura 2: Obtenção do Modelo de Carga Não-Seqüencial

As etapas para obtenção do modelo Markoviano agregado, a partir da curva cronológica de carga, são apresentadas no fluxograma da Figura 2.

Para consideração do efeito da carga nos índices de freqüência e duração do sistema, é utilizada a função teste da LOLF para a carga proposta em (Mello et al., 1993), a ser adicionada à função teste do sistema:

$F_{L O L F}\left(x_{i}\right)= \begin{cases}0 & \text { se } x_{i} \in \Omega_{S} \\ \sum_{u=j+1}^{m k} \lambda_{j u}-\sum_{v=1}^{j-1} \frac{P\left(x_{k}=v\right)}{P\left(x_{k}=j\right)} \lambda_{v j} & \text { se } x_{i} \in \Omega_{F}\end{cases}$

onde,

$\lambda_{j u}$ - é a taxa de transição de carga de um patamar $j$ para um patamar inferior $u$;

$\lambda_{v j}$ - é a taxa de transição de carga de um patamar superior $v$ para o patamar $j$;

\subsection{Usinas Eólicas}

A modelagem do vento é o aspecto mais importante a ser tratado para que se possa incorporar usinas eólicas no estudo de confiabilidade. O enfoque principal é a caracterização do comportamento do vento como sendo um processo estocástico que possa ser tratado como um modelo Markoviano de múltiplos estados.

O comportamento do vento não pode ser caracterizado, a rigor, como um processo estacionário, visto que o vento apresenta variações sazonais que tornam inconstantes a média e o desvio padrão durante determinados períodos de tempo. Porém, em (Thomann e Barfield, 1998) é mostrado que ao se considerar um período grande de amostragem, como um ou mais anos, e não se verificar nenhuma tendência específica nas medições, é possível caracterizar o vento como sendo um processo estacionário. Isso se aplica à análise de fenômenos de longo termo, como o cálculo de índices de confiabilidade associados às probabilidades estacionárias.

\subsubsection{Modelo Seqüencial do Vento}

Para a modelagem do vento visando a SMC Seqüencial, a série temporal de vento pode ser utilizada diretamente para alimentação do modelo, salvo a necessidade de se compatibilizar o nível de discretização da simulação e da série de vento. Normalmente, a série de vento é única para uma mesma usina, independente do numero de turbinas, pois, por simplificação, é comum considerar-se que todas as turbinas estão sujeitas ao mesmo vento. Na Figura 3 é apresentada uma síntese deste modelo.

Ao se variar $t_{k}$ de 0 a última hora do ano, será percorrida toda a série temporal de vento. $\mathrm{O}$ valor da velocidade do vento será usado como entrada na característica $P(v)$ do mo-

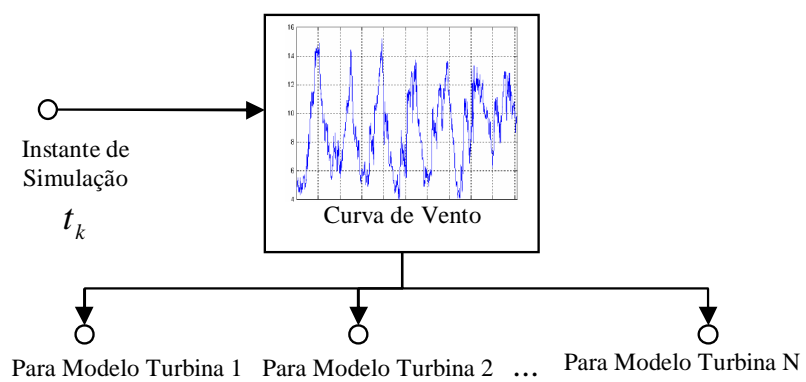

Figura 3: Modelo Cronológica do Vento 


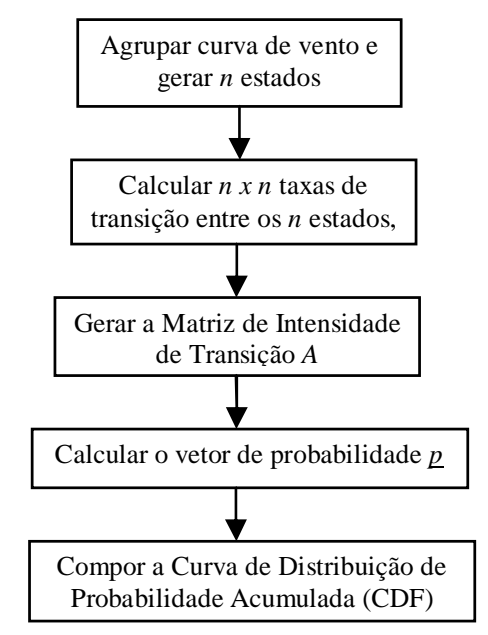

Figura 4: Obtenção do Modelo de Vento Não-Seqüencial

delo da turbina, representada na Figura 3 pelas saídas para os modelos de turbina, e resultará na série anual de geração da usina eólica.

\subsubsection{Modelo Não-Seqüencial do Vento}

A modelagem do vento visando a SMC Não-Seqüencial é feita pela representação da série de vento por um modelo Markoviano a múltiplos estados, aplicando-se as mesmas teorias e considerações utilizados para representação de cargas na SMC Não-Seqüencial. As etapas para obtenção do modelo não-seqüencial a partir da série temporal de vento são apresentadas na Figura 4.

\subsubsection{Modelo Final da Usina Eólica}

O modelo final da usina eólica deve considerar a combinação do comportamento das turbinas e do vento. Em (Leite et al., 2006a-b) é apresentado um modelo de usina eólica que obtém a representação em espaço de estados da usina pela combinação dos estados do vento e das turbinas eólicas. A Figura 5 mostra o diagrama de transição de estados deste modelo para o caso de uma turbina e quatro estados de vento, onde as transições entre o estado de operação e falha da turbina estão representadas pelas taxas de falha e reparo $\lambda$ e $\mu$, respectivamente. A velocidade do vento está agrupada nos estados I, II, III e IV e as transições entre eles estão representadas pelas taxas $\lambda_{i j}$, onde i representa o estado de vento inicial e j o estado final. A partir deste modelo, a usina pode ser representada no sistema por um modelo markoviano a múltiplos estados, como sendo uma usina com vários patamares de geração.

O modelo proposto neste trabalho não calcula explicitamente esta representação em espaço de estados da usina. O vento e

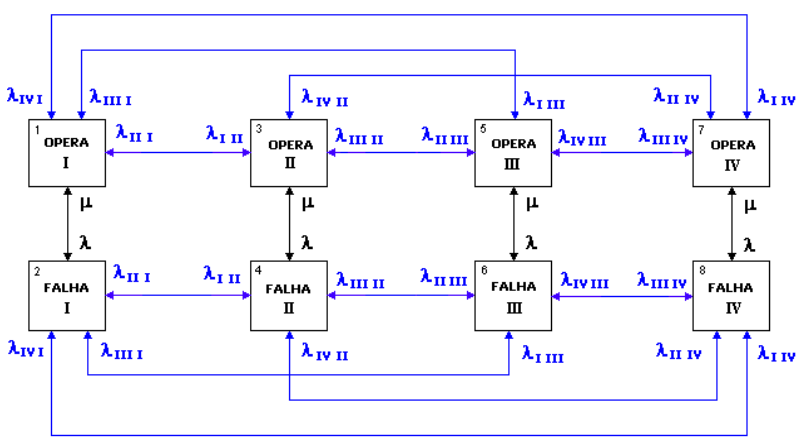

Figura 5: Modelo da usina eólica de (Leite et al., 2006a-b)

a disponibilidade das turbinas são modelados separadamente e o efeito da combinação dos dois modelos é obtido durante a simulação. Dependendo da abordagem desejada, os modelos que deverão ser combinados serão o seqüencial ou o não seqüencial de cada elemento (vento e turbina), resultando nos modelos finais mostrados, respectivamente, na Figura 6 e 7.

No modelo seqüencial, o estado do vento é determinado percorrendo-se a série temporal, enquanto que no modelo não- seqüencial ele é amostrado a partir da sua distribuição de probabilidade acumulada (CDF). Nos modelos apresentados, é considerada a possibilidade de uma usina eólica dispor de turbinas com características diferentes $\left(P_{1}(v), P_{2}(v)\right.$, etc.), embora a situação mais comum seja uma mesma característica para todas as turbinas na usina. A expressão $P_{k}(v) D_{k}$, para a $k$-ésima turbina, determina a potência gerada pela turbina. Caso a turbina esteja indisponível, $D_{k}=0$, a potência gerada é nula. Se a turbina estiver disponível, $D_{k}$ $=1$, a potência gerada dependerá do estado do vento. A potência final da usina é dada pelo somatório das potências geradas em cada turbina.

Para consideração do efeito da geração eólica nos índices de frequiência e duração do sistema, as V.A.s estado do vento e estado da turbina são consideradas no modelo de forma independente. Desta forma, para cada turbina da usina, é usada a função teste da LOLF (5), levando-se em conta as taxas de falha e reparo de cada uma. Já para o modelo do vento, é adotada a função teste para a LOLF (6), análoga à equação usada para a carga.

$$
\begin{gathered}
F_{L O L F}^{T U R B N A S}\left(x_{i}\right)= \begin{cases}0 & \text { se } x_{i} \in \Omega_{S} \\
\sum_{k \in D_{i}}^{\mu_{k}}-\sum_{k \in U_{i}}^{\lambda_{k}} & \text { se } x_{i} \in \Omega_{F}\end{cases} \\
F_{L O L F}^{V E N T O}\left(x_{i}\right)= \begin{cases}0 & \text { se } x_{i} \in \Omega_{S} \\
\sum_{u=j+1}^{m k} \lambda_{j u}-\sum_{v=1}^{j-1} \frac{P\left(x_{k}=v\right)}{P\left(x_{k}=j\right)} \lambda_{v j} & \text { se } x_{i} \in \Omega_{F}\end{cases}
\end{gathered}
$$




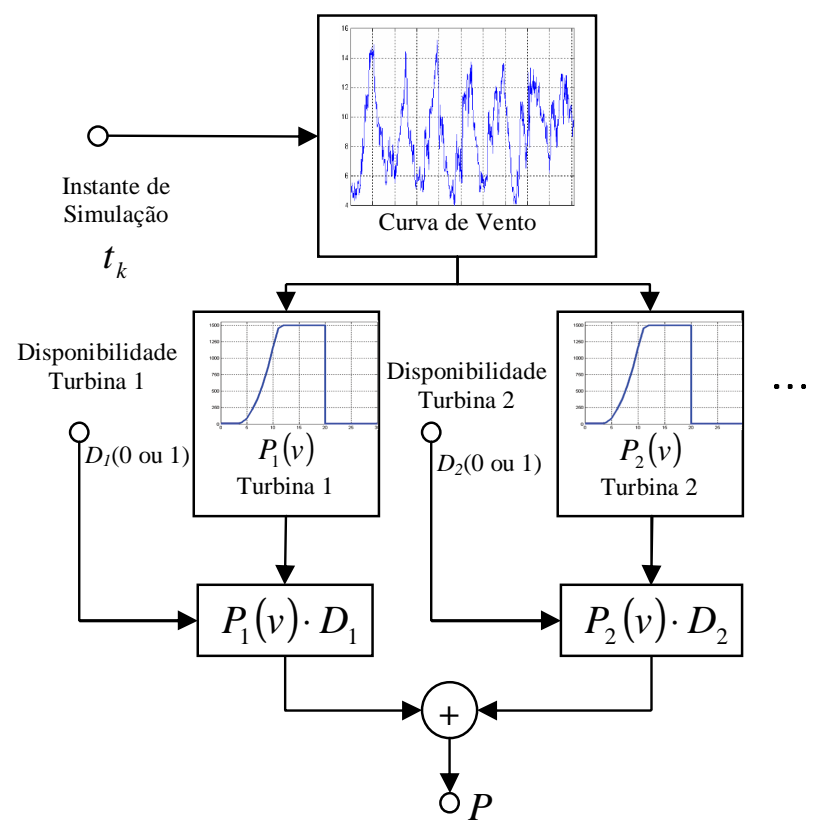

Figura 6: Modelo Seqüencial de Usina Eólica

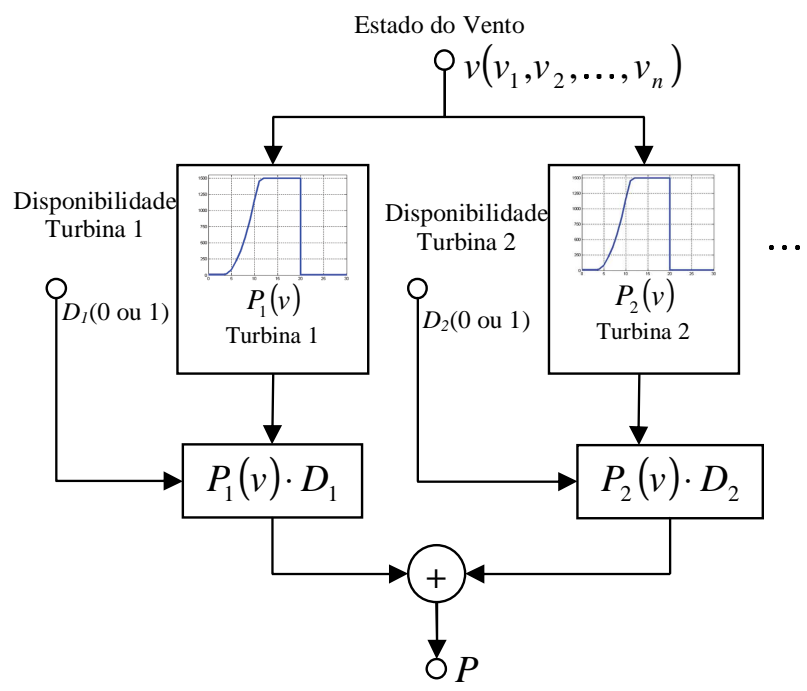

Figura 7: Modelo Não-Seqüencial de Usina Eólica

onde,

$\mu_{k}$ - é a taxa de reparo da turbina $k$;

$\lambda_{k}$ - é a taxa de falha da turbina $k$;

$\lambda_{j u}$ - é a taxa de transição do vento da velocidade $j$ para uma velocidade superior $u$; $\lambda_{v j}$ - é a taxa de transição do vento de uma velocidade superior $v$ para a velocidade $j$.

A função teste final para LOLF da usina eólica é dada por (7):

$$
F_{L O L F}^{E O L I C A}\left(x_{i}\right)=F_{L O L F}^{T U R B I N A S}\left(x_{i}\right)+F_{L O L F}^{V E N T O}\left(x_{i}\right)
$$

Observa-se que a diferença entre a formulação da função teste da carga e da geração eólica, em relação ao vento, diz respeito a consideração de quem são os estados "superiores" e "inferiores". Respeitada a hipótese de coerência, aumentar o nível de carga tende a piorar o índice da LOLF, enquanto que aumentar o nível de geração eólica tende a melhorar o índice da LOLF. Desta forma, a carga transitar para um estado "superior" consiste em diminuir o seu nível de carga, enquanto que a geração transitar para um estado "superior" consiste em aumentar o seu nível de geração.

\section{MODELO ORIENTADO A OBJETOS}

O modelo computacional desenvolvido neste trabalho foi baseado no ambiente para modelagem orientada a objetos de sistemas de potência proposto em (Manzoni, 2005). Este ambiente é constituído por um sistema de classes que permite descrever de forma flexível toda a estrutura de um sistema de potência, possibilitando que os componentes e as relações hierárquicas entre eles sejam representados o mais fiel possível para uma determinada aplicação.

Foram implementados dois tipos básicos de simuladores para a análise de confiabilidade composta: o simulador seqüencial e o não-sequiencial. Embora os dois tipos de simulação apresentem características distintas, existem alguns atributos e processos que são semelhantes para estes simuladores. Sob a ótica da MOO, fica evidente a presença de uma entidade mais geral que implementa essas características independentes do método de simulação, para a qual foi desenvolvida uma classe base chamada classe Simulador Base.

As especificidades de cada método de simulação são implementadas nas classes filhas, construídas a partir da herança direta da classe Simulador Base, e desta forma todas as funcionalidades e processos comuns são automaticamente incorporados a cada método de simulação. Esta estrutura de classes é apresentada no diagrama da Figura 8.

Na Figura 9 é apresentado um fluxograma básico com os principais processos de uma simulação Monte Carlo genérica, onde:

1. Seleção de Estados: nesta etapa os estados dos com- 


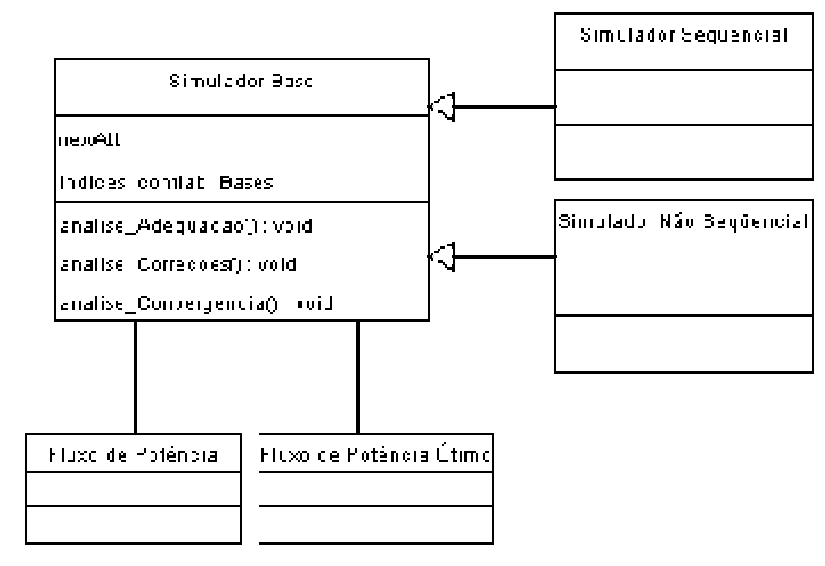

Figura 8: Diagrama de Classes dos Simuladores Monte Carlo

ponentes do sistema, níveis de geração e carga são selecionados segundo alguma enfoque específico. Como este processo depende integralmente do método de simulação, ele não pertence ao escopo da classe Simulador Base, sendo implementado no âmbito das classes filhas específicas, isto é, classe Simulador Seqüencial e classe Simulador Não-Seqüencial.

2. Análise de Adequação do Estado: nesta etapa o estado amostrado para o sistema é avaliado através de alguma função que quantifique os possíveis efeitos de violação nos limites operativos da rede, através de um fluxo de potência não linear. Este processo é independente do método de simulação e assim pertence ao escopo da classe Simulador Base.

3. Análise de Medidas Corretivas: esta etapa atua quando a análise de adequação indica que existe algum limite operativo do sistema violado, exigindo-se algumas medidas corretivas para levar o sistema para um estado operativo sem violação, pela solução de um fluxo de potência ótimo. Este processo também é independente do método de simulação e assim pertence ao escopo da classe Simulador Base.

4. Cálculo dos Índices de Confiabilidade: nesta etapa a avaliação final da adequação do estado selecionado, já considerando as medidas corretivas, é utilizada para a atualização dos índices de confiabilidade. Embora alguns desses índices sejam comuns aos dois métodos de simulação, a forma como são calculados é diferente e, desta forma, este processo é implementado no âmbito das classes filhas específicas.

5. Análise de Convergência: nesta etapa as incertezas das estimativas dos índices são analisadas segundo algum critério de convergência que decide pela finalização ou não do processo de simulação. Embora o cálculo dos índices seja função dos métodos de simulação específicos,

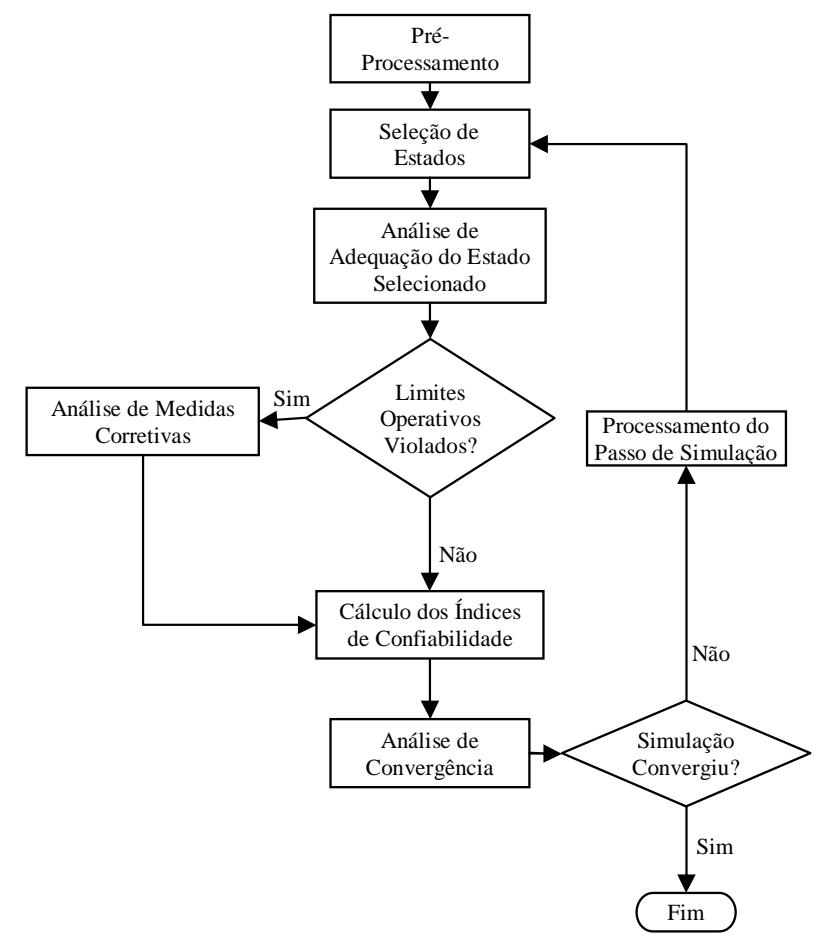

Figura 9: Fluxograma para a Simulação Monte Carlo genérica

para efeito de análise de convergência o que importa é a evolução numérica dos índices. Assim, pode-se modelar esta etapa independentemente do método de simualação, pertencendo ao escopo da classe Simulador Base.

6. Processamento do Passo de Simulação: caso o critério de convergência não tenha sido satisfeito, a simulação prossegue executando-se um avanço no passo de simulação. Este avanço é implementado de forma diferente para cada método de simualação, sendo implementado no âmbito das classes filhas específicas.

\subsection{Simulador Seqüencial}

A classe Simulador Seqüencial implementa as características dependentes do aspecto cronológico do método de simulação, afetando as etapas de Seleção de Estados, Cálculo dos Índices de Confiabilidade e Processamento do Passo de Simulação, de acordo com:

1. Seleção de Estados: nesta etapa o simulador atualiza nos modelos de componentes os estados dependentes da hora do ano. O simulador também processa a transição de estados cronológica dos componentes;

2. Cálculo dos Índices de Confiabilidade: nesta etapa os 
índices do ano de simulação são calculados e computados nas suas respectivas séries anuais;

3. Processamento do Passo de Simulação: nesta etapa a hora do ano é incrementada, sendo que na última hora do ano os índices são computados nas suas respectivas séries anuais, iniciando a primeira hora do próximo ano.

\subsection{Simulador Não-Seqüencial}

A classe Simulador Não-Seqüencial implementa as características da amostragem de espaço de estados do método de simulação, afetando as etapas de Seleção de Estados, Cálculo dos Índices de Confiabilidade e Processamento do Passo de Simulação, de acordo com:

1. Seleção de Estados: nesta etapa o simulador processa a amostragem de estados a partir das curvas CDF dos componentes;

2. Cálculo dos Índices de Confiabilidade: nesta etapa os índices são atualizados de acordo com o resultado da adequação;

3. Processamento do Passo de Simulação: nesta etapa o contador de estados simulados é incrementado.

\section{RESULTADOS}

Foram estudados vários casos baseados no sistema IEEERTS (IEEE, 1979), com algumas modificações de acordo com os objetivos específicos de cada estudo. Os casos estudados visam analisar os seguintes aspectos:

- Validação do modelo computacional;

- Equivalência entre os enfoques seqüencial e nãoseqüencial;

- Impacto da consideração da curva de carga;

- Impacto da presença de usinas eólicas.

Na Tabela 1 é apresentado um resumo dos casos simulados.

Tabela 1: Casos Simulados

O Caso 1 consitiu em avaliar o sistema RTS conforme apresentado em (IEEE, 1979), por representar uma referência para validação do modelo. Os custos unitário de corte de carga foram baseados em (Wenyuan e Billinton, 1993). Posteriormente foram realizadas as simulações de real interesse,
Tabela 1: Casos Simulados

\begin{tabular}{|c|c|c|}
\hline Caso & $\begin{array}{c}\text { Representação } \\
\text { Carga }\end{array}$ & $\begin{array}{c}\text { Representação } \\
\text { Geração }\end{array}$ \\
\hline 1 & Patamar Único & Convencional \\
\hline 2 & $\begin{array}{c}\text { Curva de } \\
\text { Carga 120\% }\end{array}$ & Convencional \\
\hline 3 & $\begin{array}{c}\text { Curva de } \\
\text { Carga 120\% }\end{array}$ & $\begin{array}{c}\text { Convencional e Eólica } \\
150 M W\end{array}$ \\
\hline
\end{tabular}

com a representação de curva de carga e das usinas eólicas. No Caso 2, foi avaliada a influência da curva de carga, considerando um nível de $120 \%$ em relação à carga nominal do Caso 1. No Caso 3, foi avaliada a influência da inserção de uma usina eólica de $150 \mathrm{MW}$ no sistema, mantendo a representação da mesma curva de carga do Caso 2. Para todas as simulações, foi considerado como critério de parada um coeficiente de variação de 5\% nos índices de confiabilidade. Os resultados foram obtidos em um computador Pentium-IV 2.5MHz com 512MB de memória RAM.

\subsection{Caso 1 - Carga e Geração Invariantes}

O sistema RTS foi analisado utilizando-se o simulador seqüencial e o não-sequencial. Os índices calculados são apresentados na Tabela 2, para o sistema, e na Tabela 3, para a EPNS das barras com carga. Os valores entre parenteses representam os coeficientes de variação dos índices.

Destaca-se que a maior parte do tempo de processamento, aproximadamente $78 \%$ do total, está na execução do fluxo de potência ótimo, principalmente pelo fato de não ter sido ainda implementada qualquer técnica de otimização do código visando a eficiência computacional.

Observa-se, pelos índices calculados por barra, que o corte de carga respeitou corretamente o critério adotado de custo de corte, quanto maior o custo, menor o corte. A barra 2 apresenta a quantidade mais significativa de corte, enquanto que a barra 13 apresenta o menor nível de corte. Observa-se que os erros relativos entre os índices calculados pela SMC Não-Seqüencial e pela SMC Seqüencial, tanto para o sistema como para as barras individuais, são bastante pequenos e sempre menores que a tolerância especificada para o coeficiente de variação.

Este caso também foi simulado usando o programa comercial NH2, desenvolvido pelo CEPEL (CEPEL, 2006). Os índices LOLP, LOLF e LOLD obtidos foram 11.22\%, 25.34 oc/ano e 39.14 horas, respectivamente, todos com erro relativo em relação aos calculados pelo modelo desenvolvido dentro da tolerância especificada para o coeficiente de variação. 
Tabela 2: Índices do Sistema - Caso 1

\begin{tabular}{|c|c|c|c|}
\hline Índice & Sequencial & $\begin{array}{c}\text { Não- } \\
\text { Sequencial }\end{array}$ & $\begin{array}{l}\text { Erro } \\
\text { Rela- } \\
\text { tivo }\end{array}$ \\
\hline LOLP & $11.1 \%(0.033)$ & $\begin{array}{c}11.04 \% \\
(0.01)\end{array}$ & $0.55 \%$ \\
\hline EPNS & $\begin{array}{c}19.03 \mathrm{MW} \\
(0.05)\end{array}$ & $\begin{array}{c}18.92 \mathrm{MW} \\
(0.046)\end{array}$ & $0.58 \%$ \\
\hline EENS & $166702 \mathrm{MWh}$ & $\begin{array}{l}165739 \\
\text { MWh }\end{array}$ & $0.58 \%$ \\
\hline LOLF & $\begin{array}{c}23.10 \mathrm{oc} / \mathrm{ano} \\
(0.025)\end{array}$ & $\begin{array}{c}24.15 \text { oc/ano } \\
(0.05)\end{array}$ & $4.5 \%$ \\
\hline LOLD & 42.09 horas & 40.04 horas & $4.8 \%$ \\
\hline $\begin{array}{c}\text { Estados } \\
\text { Simulados }\end{array}$ & $\begin{array}{c}919800(105 \\
\text { anos) } \\
(105 \text { anos })\end{array}$ & 6136 & \\
\hline $\begin{array}{c}\text { Tempo } \\
\text { Total }\end{array}$ & $43 \mathrm{~min}$ & $8 \mathrm{~min}$ & \\
\hline
\end{tabular}

Tabela 3: EPNS por Barra com Carga - Caso 1

\begin{tabular}{|c|c|c|c|c|c|c|c|}
\hline Barra & $\begin{array}{c}\text { EPNS(MW) } \\
\text { Seqüencial }\end{array}$ & $\begin{array}{c}\text { EPNS(MW) } \\
\text { Não-Seq. }\end{array}$ & $\begin{array}{c}\text { Erro } \\
\text { Rela- } \\
\text { tivo }\end{array}$ & Barra & $\begin{array}{c}\text { EPNS(MW) } \\
\text { Seqüencial }\end{array}$ & $\begin{array}{c}\text { EPNS(MW) } \\
\text { Não-Seq. }\end{array}$ & $\begin{array}{c}\text { Erro } \\
\text { Rela- } \\
\text { tivo }\end{array}$ \\
\hline 1 & 1.12 & 1.11 & $0.10 \%$ & 10 & 1.07 & 1.05 & $1.33 \%$ \\
\hline 2 & 1.59 & 1.59 & $0.12 \%$ & 13 & 0.78 & 0.79 & $1.68 \%$ \\
\hline 3 & 1.16 & 1.15 & $0.83 \%$ & 14 & 0.88 & 0.86 & $1.53 \%$ \\
\hline 4 & 1.53 & 1.53 & $0.61 \%$ & 15 & 0.88 & 0.86 & $1.53 \%$ \\
\hline 5 & 1.20 & 1.19 & $0.63 \%$ & 16 & 1.07 & 1.06 & $0.35 \%$ \\
\hline 6 & 1.21 & 1.20 & $0.59 \%$ & 18 & 0.87 & 0.85 & $1.61 \%$ \\
\hline 7 & 1.20 & 1.19 & $0.63 \%$ & 19 & 0.90 & 0.89 & $0.21 \%$ \\
\hline 8 & 1.43 & 1.43 & $0.29 \%$ & 20 & 1.15 & 1.15 & $0.03 \%$ \\
\hline 9 & 1.03 & 1.02 & $0.56 \%$ & & & & \\
\hline
\end{tabular}

\subsection{Caso 2 - Carga Variante}

O sistema RTS apresenta um nível de confiabilidade muito alta (LOLP de $0,73 \%$ ) quando se considera a representação da curva de carga proposta em (IEEE, 1979). Os efeitos da incorporação de usinas eólicas a este sistema não seriam bem observáveis, ainda mais que a EPNS pouco maior que $1 \mathrm{MW}$. Devido a isso, foi aplicado um aumento de $20 \%$ nos valores nominais de carga, visando obter índices de confiabilidade mais severos. Como o objetivo não era estressar a transmissão, foi aplicado um aumento igual de $20 \%$ nos limites de transmissão do sistema. Os índices do sistema calculados para este caso são apresentados na Tabela 4.

Tabela 4: Índices do Sistema - Caso 2

\begin{tabular}{|c|c|c|c|}
\hline Índice & Sequencial & $\begin{array}{c}\text { Não- } \\
\text { Sequencial }\end{array}$ & $\begin{array}{c}\text { Erro } \\
\text { Rela- } \\
\text { tivo }\end{array}$ \\
\hline LOLP & $8.20 \%(0.033)$ & $8.29 \%(0.01)$ & $1.09 \%$ \\
\hline EPNS & $\begin{array}{c}19.02 \mathrm{MW} \\
(0.05)\end{array}$ & $\begin{array}{c}18.42 \mathrm{MW} \\
(0.05)\end{array}$ & $3.15 \%$ \\
\hline EENS & $\begin{array}{c}166615.2 \\
\text { MWh }\end{array}$ & $\begin{array}{c}161359.2 \\
\text { MWh }\end{array}$ & $3.15 \%$ \\
\hline LOLF & $\begin{array}{c}103.2 \text { oc/ano } \\
(0.02)\end{array}$ & $\begin{array}{c}102.27 \\
\text { oc/ano } \\
(0.042)\end{array}$ & $0.9 \%$ \\
\hline LOLD & 6.97 horas & 7.1 horas & $1.9 \%$ \\
\hline $\begin{array}{c}\text { Estados } \\
\text { Simulados }\end{array}$ & $\begin{array}{c}271560(31 \\
\text { anos })\end{array}$ & 7710 & - \\
\hline $\begin{array}{c}\text { Tempo } \\
\text { Total }\end{array}$ & $1 \mathrm{~h} 4$ min & 11 min & - \\
\hline
\end{tabular}

Observa-se que todos os índices possuem erros relativos baixos, o que demonstra a equivalência entre os resultados obtidos com as duas simualações. Para os índices calculados por barra, o corte de carga também respeitou o critério adotado de custo de corte.

Na Figura 10 é apresentado o histograma do percentual acumulado dos estados de corte de determinados valores. Este gráfico permite uma interpretação interessante a respeito do sistema. A princípio, desconsiderando as restrições físicas de transmissão, isto é, assumindo que a transmissão não falha e tem capacidade infinita, é possível estimar a geração adicional necessária ao sistema para se atingir um determinado nível de confiabilidade. Por exemplo, assumindo que se deseje melhorar a LOLP de $8 \%$ para 5\%, deveria-se aumentar a capacidade de geração em aproximadamente $150 \mathrm{MW}$, o que corresponde a eliminar uma quantidade de $3 \%$ dos estados de corte. É claro que esta hipótese, a rigor, ainda considera que a geração adicional nunca falharia. Nas mesmas considerações, adicionar $700 \mathrm{MW}$ de geração ao sistema levaria a probabilidade de falha para quase zero.

\subsection{Caso 3 - Carga Variante e Geração Eólica}

Foi incorporada ao sistema do Caso 2 uma fazenda eólica com capacidade total de aproximadamente $150 \mathrm{MW}$, constituída de 84 turbinas de capacidade de $1.8 \mathrm{MW}$. A usina foi alocada na barra 10 do sistema. Os dados da usina eólica e da série de vento foram extraídos de (Leite et al., 2006a-b), 


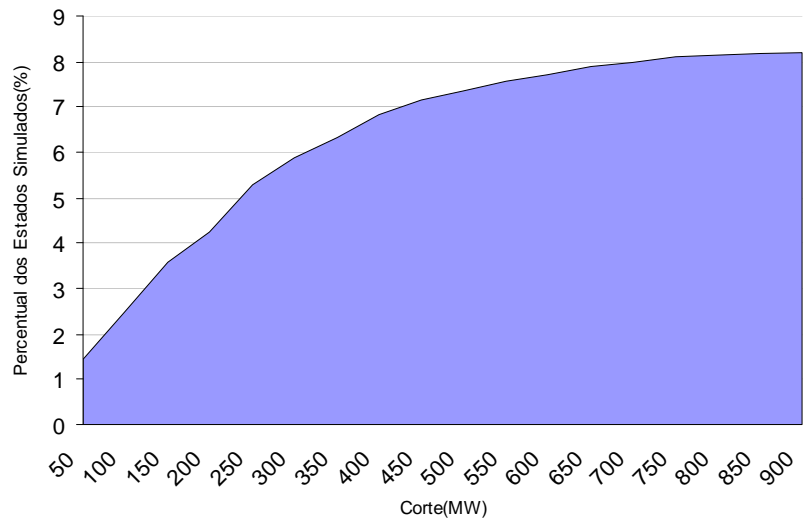

Figura 10: Percentual Acumulado dos Estados de Corte

sendo as taxas de falha e de reparo das turbinas iguais a $4 \mathrm{e}$ 90 ocorrências/ano, respectivamente. Os índices calculados são apresentados na Tabela 5 para o sistema, com o vento representado por todos as 165 valores de velocidade distintos.

Tabela 5: Índices do Sistema - Caso 3

\begin{tabular}{|c|c|c|c|}
\hline Índice & Sequencial & $\begin{array}{c}\text { Não- } \\
\text { Sequencial }\end{array}$ & $\begin{array}{c}\text { Erro } \\
\text { Rela- } \\
\text { tivo }\end{array}$ \\
\hline LOLP & $7.42 \%(0.032)$ & $\begin{array}{c}7.35 \% \\
(0.008)\end{array}$ & $0.9 \%$ \\
\hline EPNS & $\begin{array}{c}16.74 \mathrm{MW} \\
(0.05)\end{array}$ & $\begin{array}{c}16.57 \mathrm{MW} \\
(0.023)\end{array}$ & $1.0 \%$ \\
\hline EENS & $\begin{array}{c}146642.4 \\
\text { MWh }\end{array}$ & $\begin{array}{c}145153 \\
\text { MWh }\end{array}$ & $1.0 \%$ \\
\hline LOLF & $\begin{array}{c}136 \text { oc/ano } \\
(0.03)\end{array}$ & $\begin{array}{c}138.2 \text { oc/ano } \\
(0.05)\end{array}$ & $1.6 \%$ \\
\hline LOLD & $4.78 \mathrm{horas}$ & 4.66 horas & $2.7 \%$ \\
\hline $\begin{array}{c}\text { Estados } \\
\text { Simulados }\end{array}$ & $\begin{array}{c}306600(35 \\
\text { anos })\end{array}$ & 40107 & - \\
\hline $\begin{array}{c}\text { Tempo } \\
\text { Total }\end{array}$ & $22 \mathrm{~h} 17 \mathrm{~min}$ & $57 \mathrm{~min}$ & - \\
\hline
\end{tabular}

A incorporação da usina eólica melhorou a confiabilidade do sistema, provocando a queda da LOLP de $8.20 \%$ para $7.42 \%$ e da EPNS de 19.02 MW para 16.74 MW. A LOLF piorou, aumentando de 103 oc/ano para 136 oc/ano. A piora da LOLF se deve ao fato de que alguns estados contíguos de corte, que contam como uma única interrupção no Caso 2, passam a ser intercalados por um estado de sucesso, implicando no aumento do número de interrupções. A presença da geração eólica, dada a dinâmica do vento, faz com que o sistema saia da condição de falha em um determinado ins-

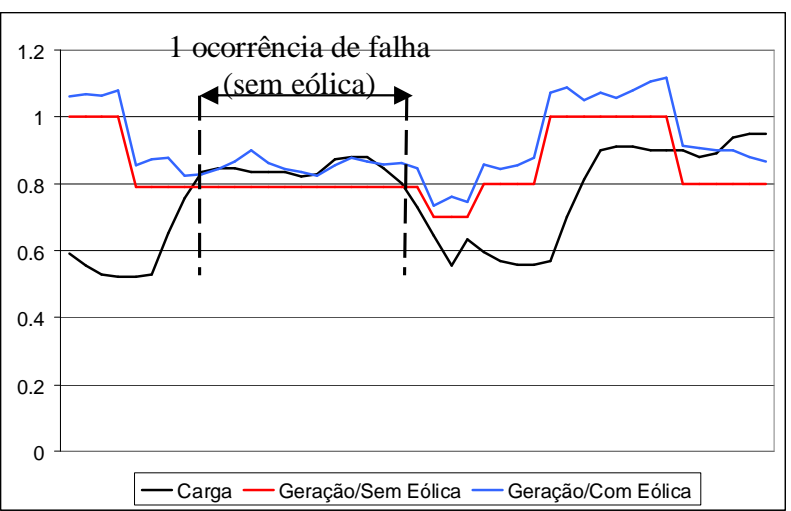

Figura 11: Efeito da Geração Eólica na Freqüência de Corte

tante, mas retorne muito rapidamente a este estado. A Figura 11 exibe um período capturado da simulação, onde são apresentados a carga total do sistema, a geração sem considerar a eólica e a geração com a eólica. O período delimitado mostra bem este efeito. $\mathrm{O}$ cruzamento da carga com a geração convencional contabiliza uma única falha neste período. Porém, ao se incluir a geração eólica, apesar do nível de geração aumentar, a dinâmica rápida da geração adicional resulta em vários cruzamentos entre a carga e a geração neste mesmo período, aumentado a frequiência de falhas do sistema. Por outro lado, a duração média das falhas se reduz com isso, passando de 6.97 horas para 4.78 horas.

A fim de avaliar o efeito do agrupamento da série de vento, foram simuladas três representações adicionais para o vento: com 3 e 10 agrupamentos de vento obtidos pela técnica kmeans, e considerando que o vento fosse constante no valor que faz a usina sempre gerar o máximo, a menos das falhas nas turbinas, que foram mantidas. Os índices calculados pela SMC Não-Sequencial estão mostrados na Tabela 6.

Observa-se que os índices calculados são bastante semelhantes para os diferentes agrupamentos do vento. A diferença mais acentuada está no número de estados necessários para a convergência, o que repercute no tempo de simulação.

Os índices obtidos para o caso de vento constante apresentam o sistema bem mais confiável do que nos casos da variação do vento, como era esperado, uma vez que a grande incerteza das usinas eólicas está relacionada ao comportamento do vento e não às falhas nas turbinas. Observa-se que a LOLP obtida para a análise de vento constante, de aproximadamente 5\%, está de acordo com a análise feita no Caso 2 , em que aumentar a capacidade de geração em $150 \mathrm{MW}$ levaria a redução de $3 \%$ na probabilidade de corte de carga. O aumento da EPNS, em relação ao caso do vento constante, é de aproximadamente $49 \%$ quando se considera a variação do vento. 
Tabela 6: Efeito do Agrupamento do Vento - Caso 3

\begin{tabular}{|c|c|c|c|}
\hline Índice & 3 Estados & 10 Estados & $\begin{array}{c}\text { Vento } \\
\text { Cons- } \\
\text { tante }\end{array}$ \\
\hline LOLP & $7.31 \%(0.003)$ & $\begin{array}{c}7.20 \% \\
(0.005)\end{array}$ & $\begin{array}{c}5.19 \% \\
(0.038)\end{array}$ \\
\hline EPNS & $\begin{array}{c}16.50 \mathrm{MW} \\
(0.028)\end{array}$ & $\begin{array}{c}16.29 \mathrm{MW} \\
(0.032)\end{array}$ & $\begin{array}{c}10.74 \\
\mathrm{MW} \\
(0.040)\end{array}$ \\
\hline EENS & $144540 \mathrm{MWh}$ & $\begin{array}{c}140948 \\
\text { MWh }\end{array}$ & $\begin{array}{c}94082.4 \\
\text { MWh }\end{array}$ \\
\hline LOLF & $\begin{array}{c}134.0 \mathrm{oc} / \mathrm{ano} \\
(0.05)\end{array}$ & $\begin{array}{c}133.5 \mathrm{oc} / \mathrm{ano} \\
(0.05)\end{array}$ & $\begin{array}{c}74.95 \\
\mathrm{oc} / \mathrm{ano} \\
(0.10)\end{array}$ \\
\hline LOLD & 4.78 horas & 4.72 horas & $\begin{array}{c}6.06 \\
\text { horas }\end{array}$ \\
\hline $\begin{array}{c}\text { Estados } \\
\text { Simulados }\end{array}$ & 28856 & 14645 & 38700 \\
\hline $\begin{array}{c}\text { Tempo } \\
\text { Total }\end{array}$ & 41 min & 19 min & 49 min \\
\hline
\end{tabular}

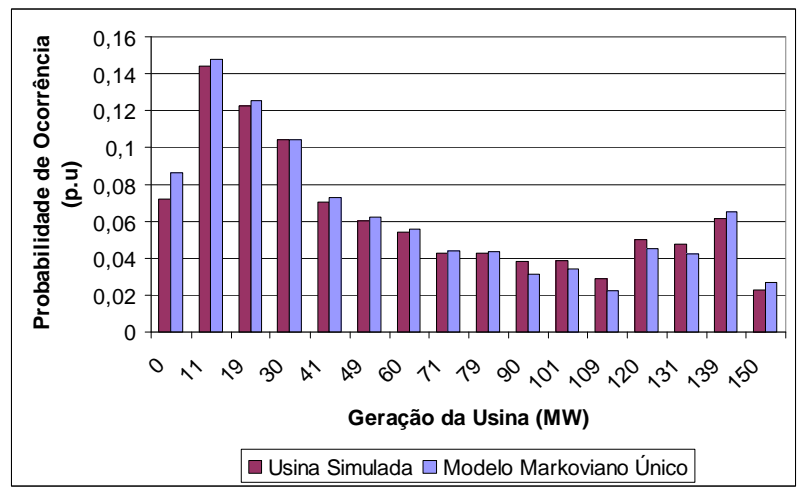

Figura 12: Comparação entre Modelo da Usina Eólica Implementado e o Apresentado em (Leite et al., 2006a-b)

Para validação do modelo de usina eólica implemento na simulação não-sequencial em relação ao modelo markoviano apresentado em (Leite et al., 2006a-b), foi gerado um histograma dos estados amostrados de geração total da usina eólica e comparado com as probabilidades dos estados de geração da mesma usina obtida pelo modelo markoviano em espaço de estados. A comparação pode ser vista na Figura 12, que mostra claramente que o modelo simulado neste trabalho tem uma grande correspondência com o modelo de usina obtido por (Leite et al., 2006a-b). Teoricamente, simulando-se uma quantidade muito grande de estados, a diferença entre os valores dos dois modelos tende a zero.

\section{CONCLUSÕES}

Este trabalho apresentou um modelo computacional flexível para avaliação de confiabilidade composta tanto no enfoque seqüencial quanto no não-seqüencial, utilizando modelagem orientada a objetos. O enfoque principal dado ao modelo foi a capacidade de representação de cargas variantes e, principalmente, de geração eólica, para a aplicação de SMC Seqüencial e Não-Seqüencial. Para a representação do modelo da usina eólica, foi adotada a separação dos modelos de turbina e de vento, evitando-se assim a necessidade de manipular um grande espaço de estados resultante da combinação dos estados do vento e de um grande número de turbinas.

A partir do modelo desenvolvido foram simulados alguns casos, sob os dois enfoques da SMC, com vistas a comparar e validar a representação dos elementos cronológicos na simulação não-seqüencial. Também foram observados os efeitos de se agrupar a curva de vento em diferentes números de estados.

Os resultados obtidos permitem concluir que, para os modelos de carga e geração adotados, a simulação não-seqüencial capturou bem o comportamento do sistema, reproduzindo os aspectos variantes no tempo com um esforço computacional muito inferior.

Para o sistema analisado, a diferença no número de estados agrupados do vento não provocou grandes alterações nos índices do sistema e tampouco no esforço computacional necessário para o processamento da simulação.

Um resultado importante observado neste trabalho foi a influência negativa que a geração eólica pode ter no índice de freqüência de corte, isto é, mesmo atuando no sentido de aumentar a capacidade do sistema, a geração eólica pode levar a um aumento da frequência de cortes de carga no sistema.

As análises considerando a simplificação de vento constante permitiram realçar o erro provocado ao se adotar modelos convencionais, sem representação da variação do vento, na análise de confiabilidade de sistema compostos com a presença de geração eólica.

\section{REFERÊNCIAS}

Billinton, R., Chen, H., Chajar, R., "A Sequential Simulation Technique for Adequacy Evaluation of Generating Systems Including Wind Energy", IEEE Transactions on Energy Conversion, v. 11, n. 4, pp. 728-734, Dec. 1996.

Billinton, R., Guang, B., "Generating Capacity Adequacy Associated with Wind Energy", IEEE Transactions on Energy Conversion, v.19, n.3, pp.641-646 Sep. 2004. 
Billinton, R., Gao, Y., "Multistate Wind Energy Conversion System Models for Adequacy Assessment of Generating Systems Incorporating Wind Energy", IEEE Transactions on Energy Conversion, v.23, n.1, Mar. 2008.

Borges, C. L. T., Falcão, D. M., Mello, J. C. O., Melo, A. C. G., "Composite Reliability Evaluation by Sequential Monte Carlo Simulation on Parallel and Distributed Processing Environments", IEEE Transactions on Power Systems, v. 16, n. 2, p. 203-209, 2001.

Cepel, Programa NH2 - Sistema Computacional para Análise Probabilística e Avaliação de Confiabilidade de Sistemas Elétricos, Versão 8.0, CEPEL, Dezembro 2006.

Foley, M., Bose, A., "Object-Oriented On-line Network Analysis", IEEE Transactions on Power Systems, vol. 10, no. 1, pp. 125-132, February 1995.

Fuerte-Esquivel, C. R., Acha, E., Tan, S. G., Rico, J., "Efficient Object Oriented Power Systems Software for the Analysis of Large-Scale Networks Containing FactsControlled Branches", IEEE Transactions on Power Systems, vol. 13, no. 2, pp. 464-472, May 1998.

Hakavik, B., Holen, A. T., "Power System Modelling and Sparse Matrix Operations Using Object-Oriented Programming", IEEE Transactions on Power Systems, vol. 9, no. 2, pp. 1045-1051, May 1994.

IEEE Task Force of APM Subcommittee, "IEEE Reliability Test System", IEEE PAS, vol. 98, no.6, pp. 2047-2054, Nov/Dez 1979.

Karki, R., Billinton, R., "Reliability/Cost Implications of PV and Wind Energy Utilization in Small Isolated Power System", IEEE Transaction Energy Conversion, vol. 16, no. 4, pp. 368-373, Dec. 2001.

Leite, A. P., Borges, C. L. T., Falcão, D. M., "Probabilistic Wind Farms Generation Model for Reliability Studies applied to Brazilian Sites", IEEE Transactions on Power Systems, Vol. 21, n. 4, p.1493-1501, 2006.

Leite, A. P., Borges, C. L. T., Falcão, D. M., "Modelagem de Usinas Eólicas para Estudos de Confiabilidade", Revista Controle \& Automação, Vol. 17, n. 2, p. 178-188, Abr-Mai-Jun 2006.

Leite da Silva, A. M., Manso, L. A. F., Mello, J. C. O., Billinton, R., "Pseudo-Chronological Simulation for Composite Reliability Analysis with Time Varying Loads", IEEE Transactions on Power Systems, Vol. 15, n. 1, pp. 73-80, Feb 2000.
Manso, L. A. F, Leite da Silva, A. M., "Modelagem de Cargas Variantes no Tempo na Avaliação de Confiabilidade Composta via Simulação Monte Carlo NãoSequencial", Revista Controle \& Automação, Vol. 15, n. 1, Jan-Fev-Mar 2004.

Manzoni, A., Silva, A. S., Decker, I. C., "Power Systems Dynamics Simulation Using Object-Oriented Programming," IEEE Transactions on Power Systems, vol. 14, no. 1, pp. 249-255, February 1999.

Manzoni, A., "Desenvolvimento de um Sistema Computacional Orientado a Objetos Para Sistemas Elétricos de Potência: Aplicação a Simulação Rápida e Análise da Estabilidade de Tensão", Tese de Doutorado, PEE - COPPE, Universidade Federal do Rio de Janeiro, Março 2005.

Mello, A. C. G., Pereira, M. V. F., Leite da Silva, A. M., "A Conditional Probability Approach to the Calculation of Frequency and Duration Indices in Composite Reliability Evaluation", IEEE Transactions on Power Systems, v. 8, n. 3, pp. 1118-1125, Agosto 1993.

Mello, J. C. O., Pereira, M. V. F., Leite da Silva, A. M., Melo, A. C. G., "Application of Chronological Load Modeling in Composite Reliability Worth Evaluation", Electric Power System Research, Vol. 40, pp. 167-174, 1997.

Melo, A. C. G., Pereira, M. V. F, Leite da Silva, A. M., "Frequency and Duration Calculations in Composite Generation and Transmission Reliability Evaluation", IEEE Transactions on Power Systems, Vol. 7, n. 2, pp. 469476, 1992.

Monticelli, A., Fluxo de Carga em Redes de Energia Elétrica, Editora Edgar Blücher, São Paulo, 1983.

Neyer, A. F, Wu, F., Imfof, K., “Object-Oriented Programming for Flexible Software: Example of a Load Flow", IEEE Transactions on Power Systems, vol. 5, no. 3, pp. 689-696, August 1990.

Pereira, M. V. F, Balu, N. J., "Composite Generation Transmission Reliability Evaluation", Proceedings of IEEE, vol.80 no.4, pp. 470-491, April 1992.

Sankarakrishnan, A., Billinton, R., "Sequential Monte Carlo Simulation for Composite Power System Reliability Analysis With Time Varying Loads", IEEE Transactions on Power Systems, Vol. 10, n. 3, pp. 1540-1545, Aug. 1995.

Singh, C., Kim, Y., "An Efficient Technique for Reliability Analysis of Power Systems Including Time Dependent Sources", IEEE Transactions on Power Systems, v. 3, n. 3, pp.1090-1096, Aug. 1988. 
Thomann, G. C., Barfield, M. J., "The Time Variation of Wind Speeds and Windfarm Power Output in Kansas", IEEE Transactions on Energy Conversion, v. 3, n. 1, pp. 44-49, March 1998.

Wenyuan, L., Billinton, R., "A Minimum Cost Assessment Method for Composite Generation and Transmission System Expansion Planning", IEEE Transactions on Power System, v.8, n.2, pp.628-635, May 1993. 